\title{
Study of Gas Separation using A Hollow Fiber Membrane with Orifices
}

\author{
Abdallah Omar Mohammad Kaddoura \\ Faculty of Engineering \\ King Abdulaziz University - Jeddah \\ Rabiulakhir 1441h - December 2019g
}

\begin{abstract}
Computational fluid dynamics was implemented and simulation was carried out to investigate the enhancement of gas separation on a binary fluid flow. $\mathrm{k}-\omega$ Baseline Reynolds stress (BSL) turbulence models was used to study the $\mathrm{CH}_{4}-\mathrm{CO}_{2}$ mixture flow characteristics and separation. The steady flow in geometry consisting of a channel, a porous layer which surrounds the channel, and supports a hollow fiber membrane (HFM). The simulation is considered as 2D. Concentration and mass flux of each mixture component will be determined using membrane selectivity and permeability of the membrane, and using local partial pressure. The effect of conical orifices is investigated along with changing their quantity and distance of one to another. The simulation is conducted on a multiple Reynolds number values. Addition of conical orifices was found to induce momentum mixing, which enhances the gas separation. However, the numerical simulation suffered a divergence issue which led to unsteady results and zero velocity on the membrane wall. Further study is recommended for orifices design, and elements leading to convergence in simulation such as mesh design and initial and boundary conditions.
\end{abstract}

\section{NOMENCLATURE}

NOMENCLATURE

\begin{tabular}{|c|c|c|c|}
\hline$C$ & concentration $\left[\mathrm{mol} / \mathrm{m}^{3}\right]$ & $U$ & average velocity $[\mathrm{m} / \mathrm{s}]$ \\
\hline$D$ & diffusion coefficient $\left[\mathrm{m}^{2} / \mathrm{s}\right]$ & $u$ & velocity vector \\
\hline$D_{2}$ & hollow fiber membrane diameter & $D_{1}$ & lumen diameter \\
\hline$K$ & permeability of porous media & $J$ & molar flux $\left[\mathrm{mol} / \mathrm{m}^{2}\right]$ \\
\hline$\omega$ & specific dissipation rate $[1 / \mathrm{s}]$ & $L$ & membrane length $[\mathrm{m}]$ \\
\hline$v$ & kinematic viscosity $\left[\mathrm{m}^{2} / \mathrm{s}\right]$ & $\ddot{P}$ & permeability $\left[\frac{\mathrm{mol}}{\mathrm{m} \mathrm{s} \mathrm{Pa}}\right]$ \\
\hline$\rho$ & density $\left[\mathrm{kg} / \mathrm{m}^{3}\right]$ & $R e$ & Reynolds number [-] \\
\hline$S h$ & Sherwood number [-] & $\alpha$ & mass selectivity [-] \\
\hline$V w$ & suction rate $[\mathrm{m} / \mathrm{s}]$ & $U$ & average velocity $[\mathrm{m} / \mathrm{s}]$ \\
\hline$d$ & orifice diameter $[\mathrm{m}]$ & $\Delta p$ & pressure difference $[\mathrm{Pa}]$ \\
\hline$k$ & turbulent kinetic energy $[\mathrm{J} / \mathrm{kg}]$ & $h m$ & mass transfer coefficient $[\mathrm{m} / \mathrm{s}]$ \\
\hline$m$ & mass flux $\left[\mathrm{kg} /\left(\mathrm{s} \mathrm{m}^{2}\right)\right]$ & $l$ & membrane thickness $[\mathrm{m}]$ \\
\hline$u$ & $x$-component of velocity $[\mathrm{m} / \mathrm{s}]$ & $p$ & pressure $[\mathrm{Pa}]$ \\
\hline$x$ & $x$ coordinate $[\mathrm{m}]$ & & \\
\hline \multicolumn{4}{|c|}{ Subscripts and Superscripts } \\
\hline$a$ and $b$ & species: $\mathrm{CO}_{2}$ or $\mathrm{CH}_{4}$ & $\mathrm{w}$ & properties at the membrane \\
\hline $\mathrm{i}$ and $\mathrm{j}$ & index notation & $\mathrm{CO}_{2}$ & properties of $\mathrm{CO}_{2}$ \\
\hline $\mathrm{CH}_{4}$ & properties of $\mathrm{CH}_{4}$ & & \\
\hline
\end{tabular}




\section{CHAPTER I \\ INTRODUCTION}

Natural gas is an important source of energy in today's world, and when extracted from underground is un-pure and mixed with other gases. The extraction process is expensive and the pipe lines included in the process are being damaged due to this un-purity thus the idea of purification and gas separation to save on oinitial and operational costs. .

Alkhamis et al. [1,2] investigated the turbulence mixing and studied its effect in Gas-Gas separation simulation in membranes numerically. membrane was modelled as functional 2D surface and Navier-stokes and mass transport equations were solved similar to the study presented here. Both models did not include porous layer Unlike the current work. Mass flux through the membrane is determined through local partial pressure of each component, membrane selectivity and permeability similar to the work here.

Alkhamis et al. [3, 4] conducted a similar 2D simulation introducing porous layer in the flow geometry, Circular channel, bounded by a porous layer which supports a HFM (like the model presented here) was studied. Similar to our and previous studies Mass flux through the membrane is determined through local partial pressure of each component, membrane selectivity and permeability, Results of around $60 \%$ improvement on the membrane performance have been reported. And the research concluded that momentum mixing will enhance gas -gas separation.

In Alreihili et. Al. [5], different flow geometry was proposed for Reynolds number values reaching 1000 on flow past an array with diamond shaped membranes in inline and staggered arrangements are concluded to be beneficial for the gas separation..

Alreihili et. Al. [6], channel bounded by upper and lower spiral wound membranes containing square spacers model was used to conduct gas separation simulation.. and as expected, enhanced the separation process. .

this study investigates the effect of momentum mixing for $\mathrm{CH}_{4}-\mathrm{CO}_{2}$ mixture flow in a channel bounded by a porous layer which support a HFM. Momentum mixing is induced using uniformly spaced conical orifices

\section{CHAPTER II \\ PROBLEM DESCRIPTION}

The geometry in this research includes an open channel bounded by a porous layer which is surrounded by a HFM as shown in Fig (1). conical orifices are placed in the channel at constant displacement from each other for the purpose of momentum mixing in both the channel and porous layer feed. The orifice diameter is $d=0.5 D_{1}$ and the spacing between two successive orifices is $s=10 d$ The flow of the $\mathrm{CH}_{4}-\mathrm{CO}_{2}$ binary mixture is illustrated in the bellow figure. $D_{1} / D_{2}$ is 0.67 and $d / D_{1}=0.5$

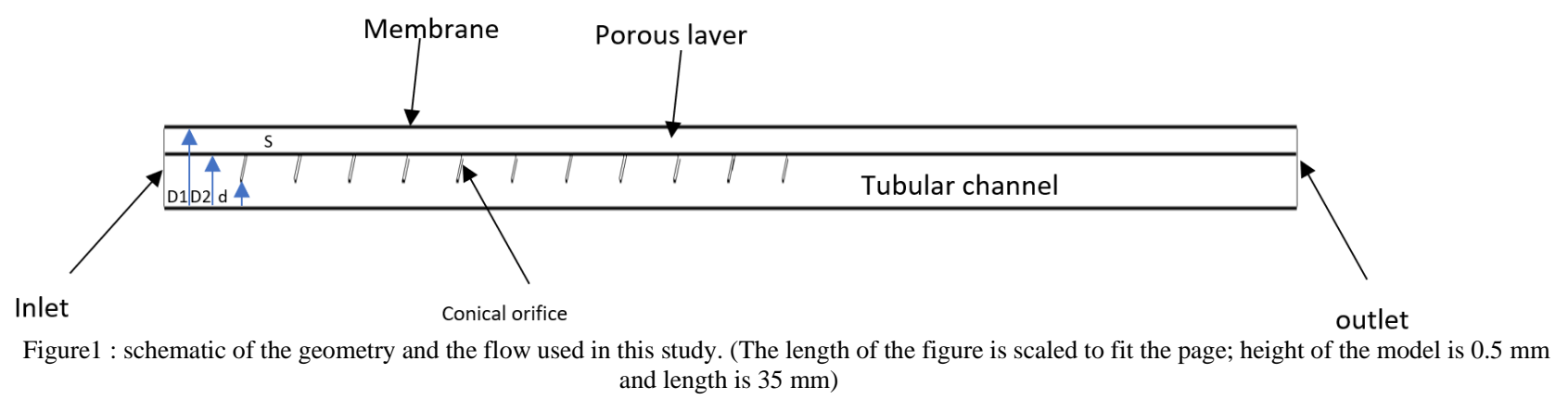

\section{CHAPTER III}

\section{GOVERNING EQUATIONS AND RESEARCH METHODOLOGY}

The governing equations that described the motion of fluid inside the feed channel are continuity equation and NavierStokes Equation in the bellow form: .

$\frac{\partial u_{j}}{\partial x_{j}}=0$

$\frac{\partial u_{i}}{\partial t}+u_{j} \frac{\partial u_{i}}{\partial x_{j}}=-\frac{1}{\rho} \frac{\partial p}{\partial x_{i}}+\frac{\partial}{\partial x_{j}}\left(\left(v+v_{T}\right) \frac{\partial u_{i}}{\partial x_{j}}\right)$

For fluid motion in laminar flow, where $i$ can be 1 or 2 as the flow is in $2 \mathrm{D}$ and $j$ is the summation index, $u_{i}$ is the fluid velocity vector, $x_{i}$ is spatial coordinates, $\rho$ is density of. $u=u_{1}$ (X direction along flow velocity), $v=u_{2}$ (y direction velocity component), $x=x_{1}$ and $y=x_{2}$. 
Suction rate through the membrane:

$V_{w}=\frac{J}{C}=\frac{P_{b} \Delta p_{t o t}}{C}\left[\alpha+(1-\alpha) N_{b}\right]$

The boundary conditions of velocity on the membrane surface demonstrates the no-slip $u=0$ and the suction rate $v=V_{w}$. However, on the orifice, no-slip no-penetration condition is applied on its surface.

The gaseous fluid mixture is assumed incompressible and carrying uniform physical properties. The behavior of the membrane performance to be studied at multiple Reynolds numbers and the effect of the conical orifices to be determined.

Inlet boundary condition were taken from the velocity and temperature component near the exit at a previous simulation similar to Alkhamis et al. [4], see figures (2-4)

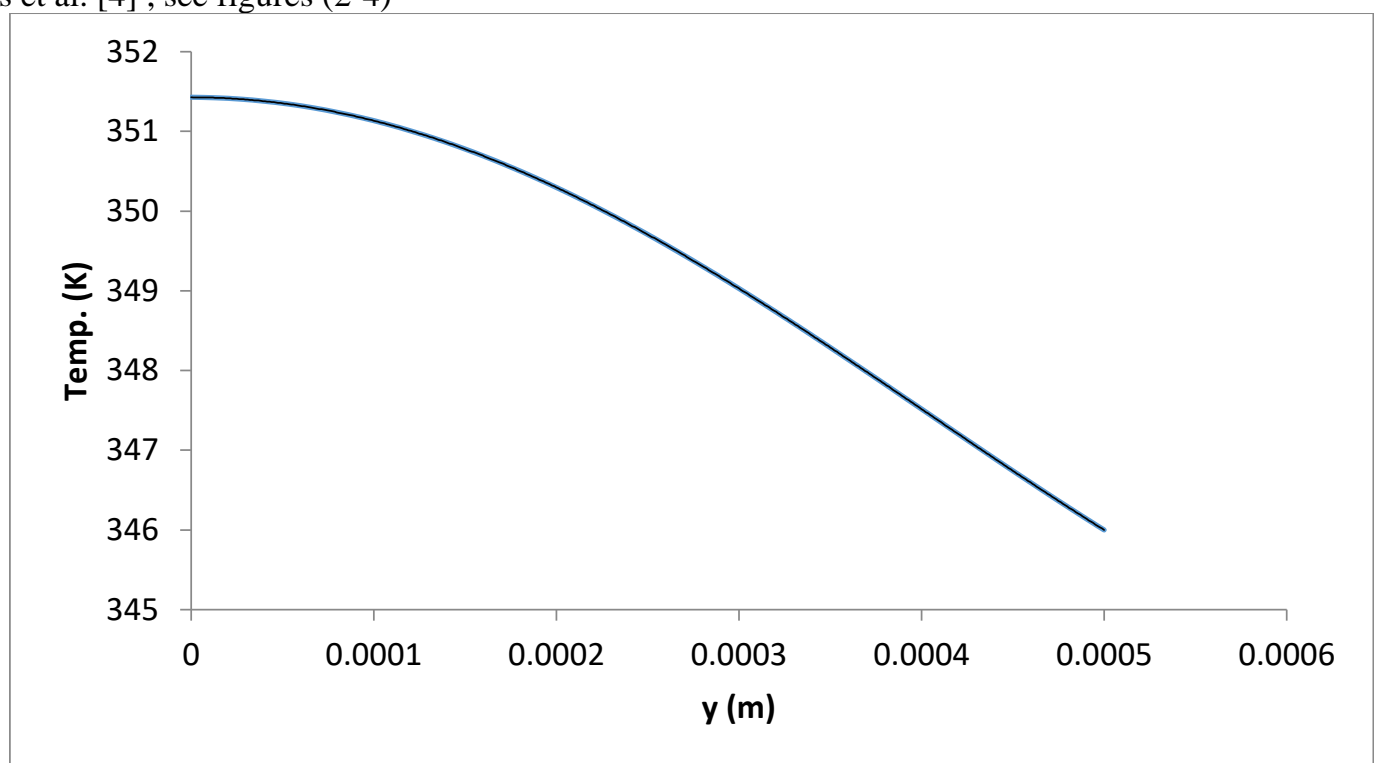

Figure (2): Initial temperature at inlet

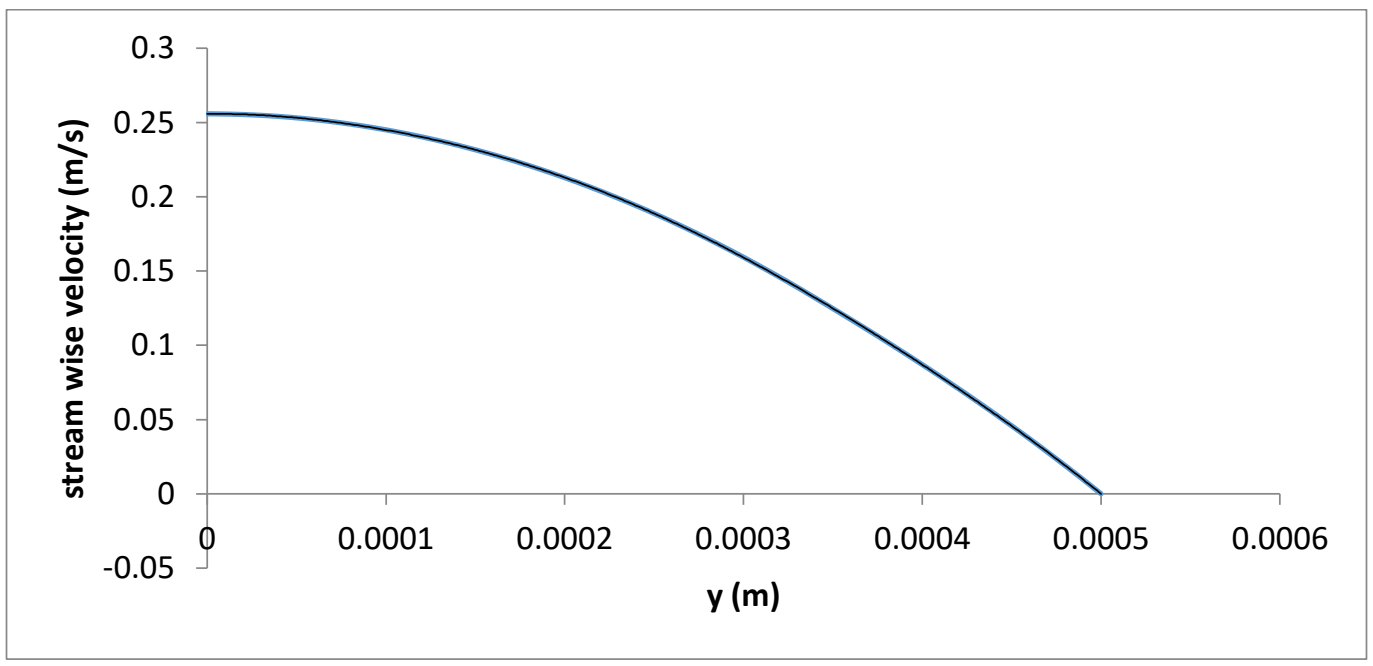

Figure (3): stream wise velocity at inlet 


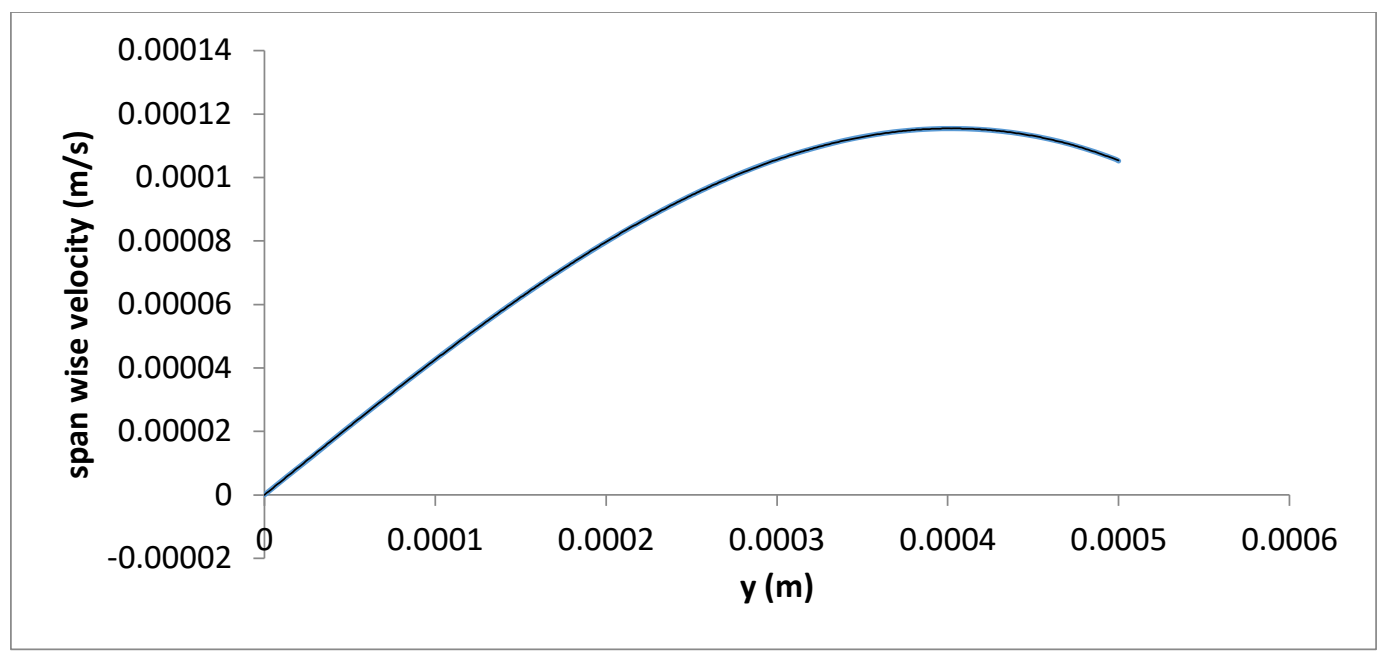

Figure (4): span wise velocity at inlet

The Sherwood number and friction factor will be calculated and compared, the local Sherwood number, and the friction factor are determined from

$h_{m}(x)=\frac{D \frac{\partial N_{a}}{\partial y}}{\left(N_{m}-N_{w}\right)}, S h=\frac{h_{m} H}{D}$ and $f=\frac{2}{\rho U^{2}} \tau_{w}$

where $h_{\mathrm{m}}$ is the mass transfer coefficient at $x, N_{\mathrm{m}}$ and $N_{\mathrm{w}}$ are the bulk mole fraction of the species " $a$ " and the mole fraction on the membrane of species " $a$ " respectively, $S h$ is the local Sherwood number, $f$ is the local friction factor and $\tau_{\mathrm{w}}$ is the wall shear stress.

The governing equations are extremely complicated for analytical work; hence numerical method of solution will be used. CFX 17.2 commercial software is used for this simulation.

\section{CHAPTER IV}

\section{RESULTS \& DISCUSSION}

The Figure (5) shows the stream wise velocity for the first conical orifices in the geometry for multiple Reynolds number values. The orifices act as obstacles and restrict the flow in the channel directing it to the porous layer and hence to the membrane. While after each orifice the flow will travel back to the channel as the resistance imposed by the orifice is no longer available till the flow reaches the next orifice. This turbulence caused by the orifices will have an impact on the concentration of the mixers and most definitely the flow through the membrane

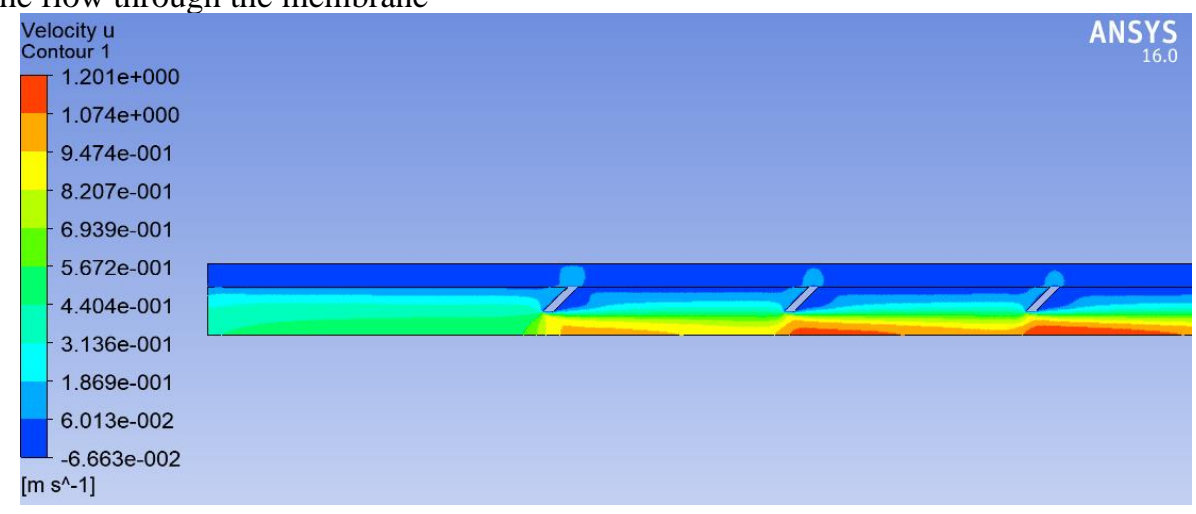




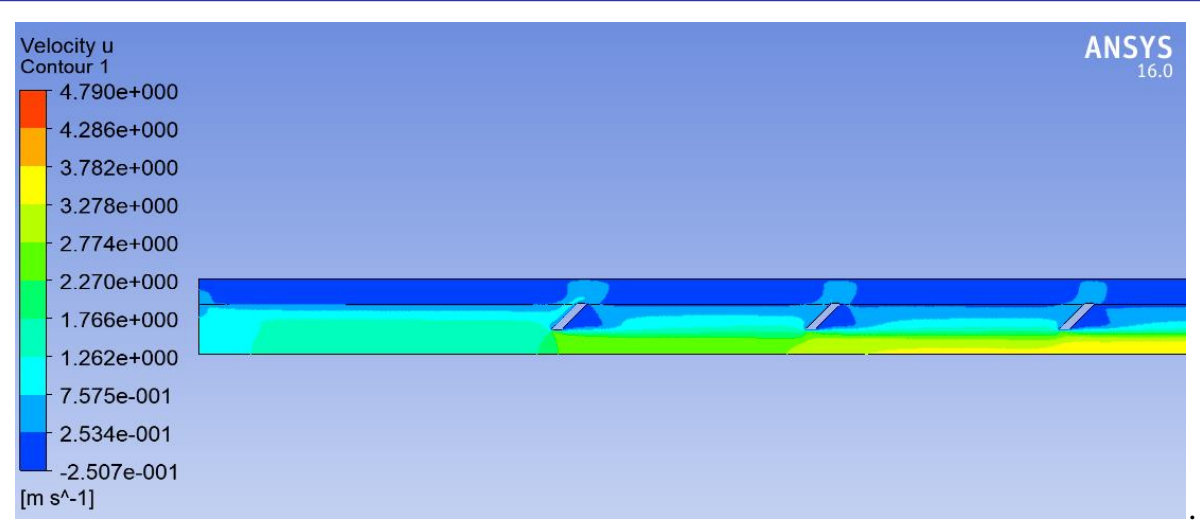

Figure 5: the stream wise component of velocity with Reynolds number $100 \& 400$ up and down respectively

The suction rate through the membrane is shown in figure (6) for Reynold numbers 100 and 400 . The suction rate through the membrane increases when Reynolds number increases. Which means that the suction rate increases at higher flow rates.
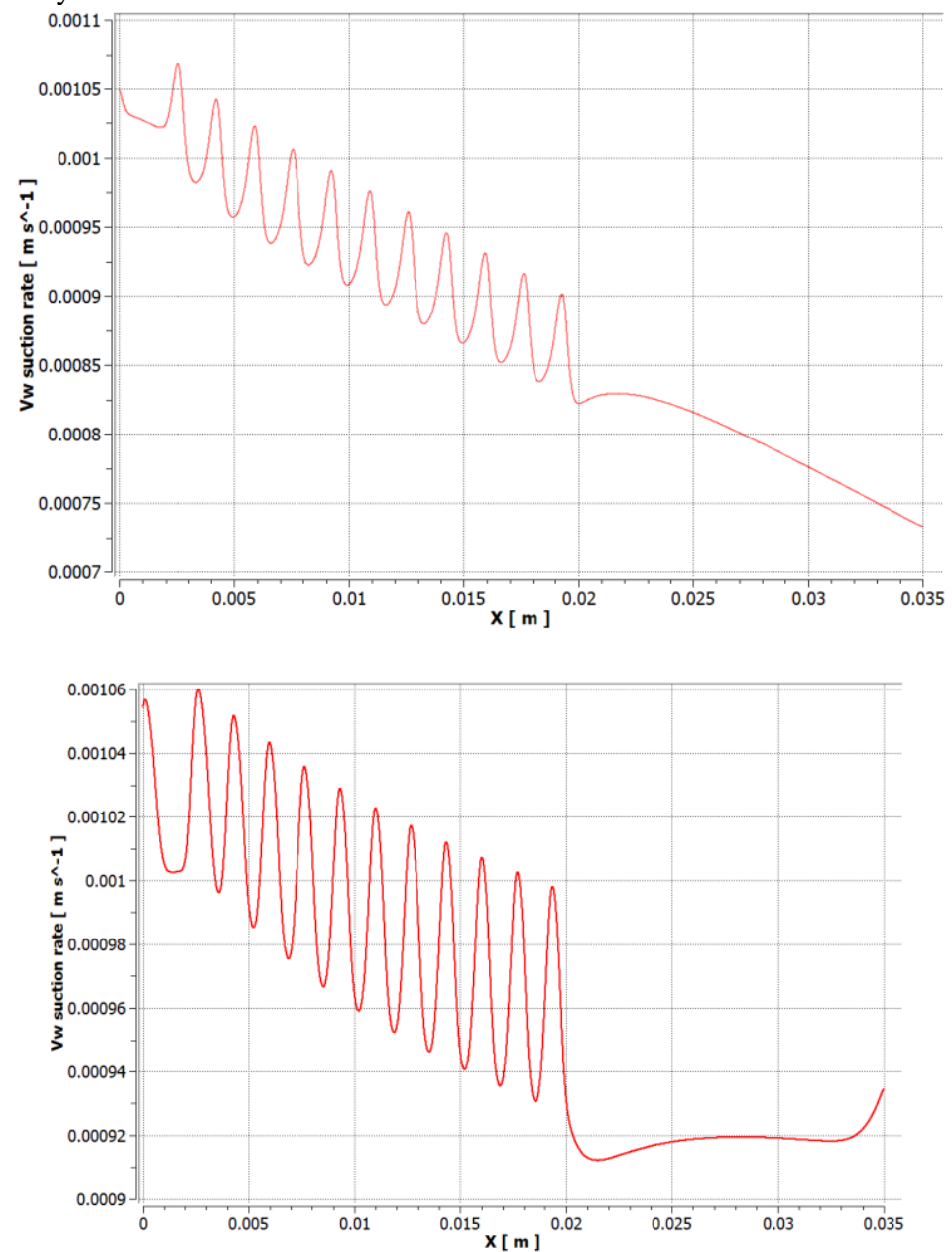

Figure 6: the suction rate through the membrane for Reynold numbers 100 and 400 respectively

After preparing the software model for simulation and starting the simulation, serious divergence issue appeared to the surface in the numerical simulation. The simulation kept crashing at the beginning of each Run giving error of divergence. The nature of error at the beginning of the simulations (usually bellow 50 iterations) implies that initial and/or boundary conditions (among input settings) to be double checked and tested for convergence. Mesh geometry, density and transitions were also a major element which usually cause divergence, in addition to other settings in the CFX software like the time step ...etc

Concerning the mesh, the mesh was divided into two main meshes, each one sketched in multiple Solid works geometries, which are uploaded to pointwise mesh software. In Pointwise, geometry was divided to structured and non-structured meshed and then the 2D meshes are uploaded into CFX, and rotationally extruded by an angle of 5 degrees. All rotations, dimensions, Orientations and directions where consistent and up to scale. Figure (7) shows the high density meshes near the membrane wall, areas of contact between porous layer and channel, and near the conical orifices, inlets and outlets are dealt with extra care, high 
mesh density and smooth transition to less density mesh were considered. After the simulation divergence the mesh was refined into higher density with higher number of cells, but same divergence issue occurred. Another trial of preparing 3D mesh on pointwise was carried but same divergence result was reported. Even mesh was tried by Ansys mesh in Ansys workbench but same result was reported which means that the mesh was not the cause of the divergence in the simulation.
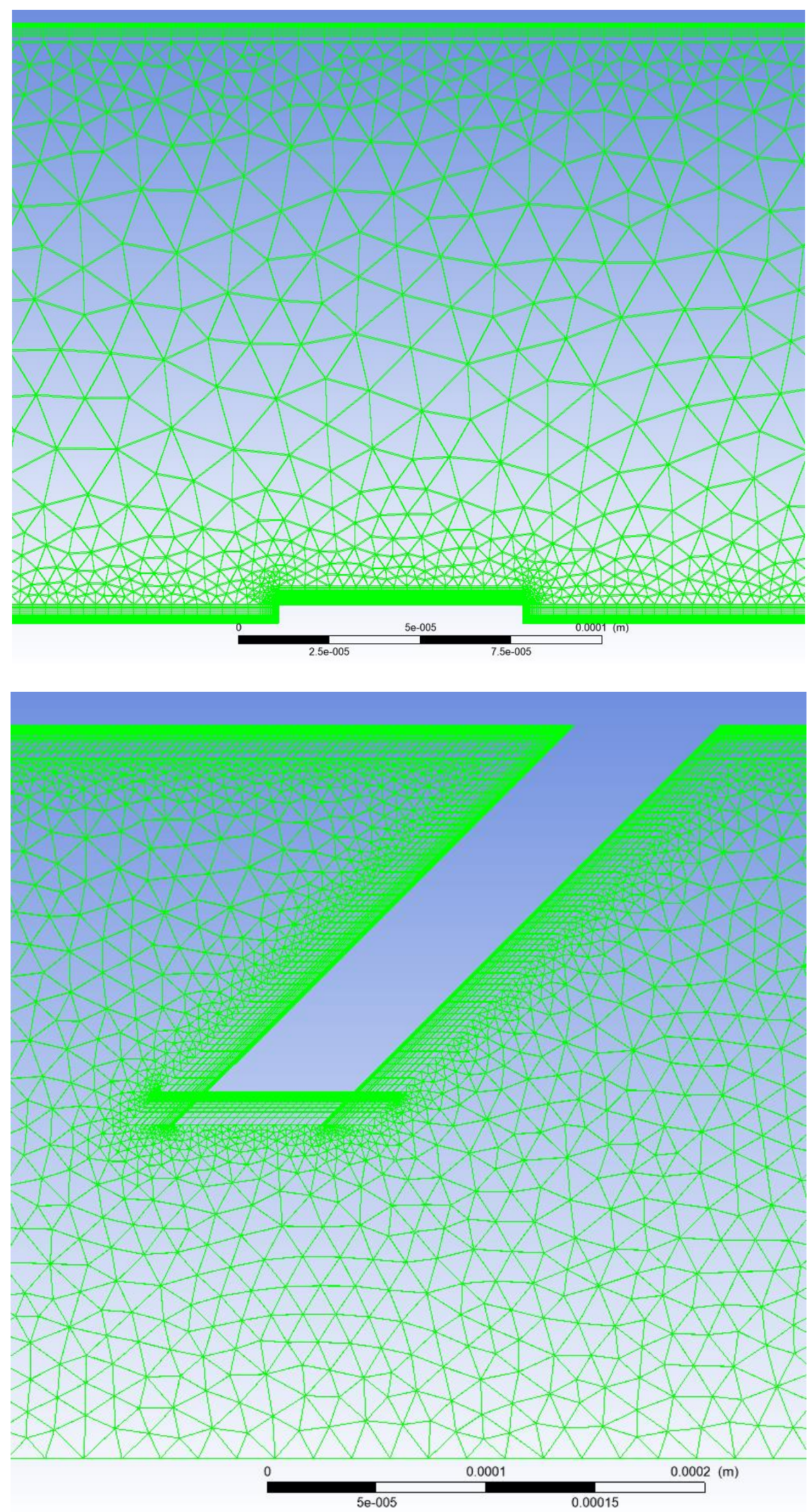

Figure 7: porous and channel meshes near the conical orifice showing areas of contact and interface. 
Concerning the initial and boundary conditions, as mentioned earlier, initial velocity components were taken near the end from previous simulation and initial conditions related to turbulence model ( $\mathrm{k}$ and epsilon) were considered zeros. Once the simulation was not convergence, shear stress transport turbulence model was simulated, the simulation gave unsteady results or result with Zero velocity on the membrane wall, which indicates that the solution was unsteady and not correct since the membrane wall was treated as permeable surface. However, the simulation still diverged at Reynolds number 200.

In the solver setting, time scales factor resulted the divergence was set to 0.85 , later it was change to a low value reaching 0.1 without mentionable use on the result, even when the residual target was set to $1 \times 10^{-4}$ instead of $1 \times 10^{-6}$ the simulation gave same answer. Time scale factor was changed to physical time scale and values around $1 \mathrm{X} 10^{-6}$ was tested but the simulation was reaching the limits of iterations without converging.

\section{CHAPTER V \\ CONCLUSION}

Computational fluid dynamics was implemented and simulation was carried out to investigate the enhancement of gas separation on a binary fluid flow. $\mathrm{k}-\omega$ Baseline Reynolds stress (BSL) turbulence models was used to study the $\mathrm{CH}_{4}-\mathrm{CO}_{2}$ mixture flow characteristics and separation. The steady flow in geometry consisting of a channel, a porous layer which surrounds the channel, and supports a hollow fiber membrane (HFM). The simulation is considered as 2D. Concentration and mass flux of each mixture component will be determined using membrane selectivity and permeability of the membrane, and using local partial pressure. The effect of conical orifices is investigated along with changing their quantity and distance of one to another. The simulation is conducted on a multiple Reynolds number values. Addition of conical orifices was found to induce momentum mixing, which enhances the gas separation. However, the numerical simulation suffered a divergence issue which led to unsteady results and zero velocity on the membrane wall. Further study is recommended for orifices design, and elements leading to convergence in simulation such as mesh design, simulation time step, and initial and boundary conditions.

\section{ACKNOWLEDGEMENTS}

I would appreciate the efforts from HPC department and the support of mechanical engineering department in king Abdul-Aziz university on their support and resources.

\section{CHAPTER VI REFERENCES}

[1] N. Alkamis, A. Anqi, D. E. Oztekin, A. Alsaiari, A. Oztekin, GAS SEPARATION USING A MEMBRANE, International Mechanical Engineering Congress and Exposition, IMECE2013.

[2] N. Alkamis, D. E. Oztekin,A. Anqi, A. Alsaiari, A. Oztekin, Numerical study of gas separation using a membrane, International Journal of Heat and Mass Transfer.80(1) (2015) 835-843

[3] N. Alkamis, A. Anqi, D. E. Oztekin, A. Alsaiari, A. Oztekin, GAS SEPARATION USING A MEMBRANE, International Mechanical Engineering Congress and Exposition, IMECE2014.

[4] N. Alkamis, A. Anqi, A. Oztekin, Computational study of gas separation using a hollow fiber membrane, International Journal of Heat and Mass Transfer.89(1) (2015) 749-759

[5] M. Alrehili, M. Usta, N. Alkamis, A.E. Anqi. A. Oztekin, Flows past arrays of hollow fiber membranes - Gas separation. (97)(1) (2016) 400-411

[6] M. Alrehili, M. Usta, N. Alkamis, A.E. Anqi. A. Oztekin, Gas Separation by Using Spiral Wound Membrane - ASME 2015 International Mechanical Engineering Congress \& Exposition. IMECE2015 